\title{
Improved outcome of pediatric patients with acute megakaryoblastic leukemia in the AML-BFM 04 trial
}

\author{
Jana Schweitzer ${ }^{1}$ - Martin Zimmermann ${ }^{1}$ - Mareike Rasche ${ }^{2}$ - Christine von Neuhoff ${ }^{2}$. \\ Ursula Creutzig $^{1} \cdot$ Michael Dworzak $^{3}$ • Dirk Reinhardt ${ }^{2} \cdot$ Jan-Henning Klusmann $^{1}$
}

Received: 24 March 2015 / Accepted: 14 April 2015 / Published online: 28 April 2015

(C) The Author(s) 2015. This article is published with open access at Springerlink.com

\begin{abstract}
Despite recent advances in the treatment of children with acute megakaryoblastic leukemia (AMKL) using intensified treatment protocols, clear prognostic indicators, and treatment recommendations for this acute myeloid leukemia (AML) subgroup are yet to be defined. Here, we report the outcome of 97 pediatric patients with de novo AMKL (excluding Down syndrome [DS]) enrolled in the prospective multicenter studies AML-BFM 98 and AML-BFM 04 (1998-2014). AMKL occurred in $7.4 \%$ of pediatric AML cases, at younger age (median 1.44 years) and with lower white blood cell count (mean $16.5 \times 10^{9} / \mathrm{L}$ ) as compared to other AML subgroups. With $60 \pm 5 \%$, children with AMKL had a lower 5-year overall survival (5-year OS; vs. $68 \pm 1 \%$, $\left.P_{\log \text { rank }}=0.038\right)$. Yet, we achieved an improved 5-year OS in AML-BFM 04 compared to AML-BFM 98 ( $70 \pm 6 \%$ vs. $45 \pm$ $\left.8 \%, P_{\log \text { rank }}=0.041\right)$. Allogeneic hematopoietic stem cell transplantation in first remission did not provide a significant survival benefit (5-year OS $70 \pm 11 \%$ vs. $63 \pm 6 \%$; $P_{\text {Mantel- }}$
\end{abstract}

Dirk Reinhardt and Jan-Henning Klusmann contributed equally to this work.

Electronic supplementary material The online version of this article (doi:10.1007/s00277-015-2383-2) contains supplementary material, which is available to authorized users.

Jan-Henning Klusmann

klusmann.jan-henning@mh-hannover.de

1 Department of Pediatric Hematology and Oncology, Hannover Medical School, Hannover, Germany

2 Ped. Hematology and Oncology, Pediatrics III, University Hospital Essen, Essen, Germany

3 St. Anna Children's Hospital and Children's Cancer Research Institute, Department of Pediatrics, Medical University of Vienna, Vienna, Austria
Byar $=0.85)$. Cytogenetic data were available for $n=78$ patients. AMKL patients with gain of chromosome 21 had a superior 5-year OS $\left(80 \pm 9 \%, P_{\log \text { rank }}=0.034\right)$, whereas translocation $t(1 ; 22)(\mathrm{p} 13 ; \mathrm{q} 13)$ was associated with an inferior 5 year event-free survival $\left(38 \pm 17 \%, P_{\log \text { rank }}=0.04\right)$. However, multivariate analysis showed that treatment response (bone marrow morphology on day 15 and 28) was the only independent prognostic marker ( $\mathrm{RR}=4.39 ; 95 \% \mathrm{CI}, 1.97-9.78)$. Interestingly, GATA1-mutations were detected in six patients (11\%) without previously known trisomy 21 . Thus, AMKL (excluding DS) remains an AML subgroup with inferior outcome. Nevertheless, with intensive therapy regimens, a steep increase in the survival rates was achieved.

Keywords Acute megakaryoblastic leukemia $\cdot$ AMKL $\cdot$ FAB M7 · AML-BFM 98 - AML-BFM 04

\section{Introduction}

Acute megakaryoblastic leukemia (AMKL), M7 according to the French-American-British (FAB) classification [1], is a specific subtype of acute myeloid leukemia (AML) that can be clearly distinguished from other subtypes because of its biological and clinical characteristics. Morphologically, AMKL blasts typically show cytoplasmic blebs, which are immunophenotypically positive for CD41, CD42b, and CD61. Myelofibrosis and manifestation as extramedullary disease are common features of AMKL and often hamper diagnosis.

AMKL occurs predominantly in childhood and particularly in children with Down syndrome (DS) [2-5], accounting for approximately $10 \%$ of pediatric AML cases. In contrast to DS-AMKL ( $\sim 0 \% \%$ survival), non-DS-AMKL is an AML subgroup associated with poor prognosis. For some studies, 
survival rates of only $14-36 \%$ were reported [5-7], whereas for other studies, survival rates of $50-70 \%$ were reported $[6$, 8]. Within the AML-BFM studies, the poor outcome of nonDS-AMKL in the AML-BFM 87 trial (5-year event-free survival [5-year EFS] $11 \pm 7 \%$, 5-year overall survival [5-year OS] $21 \pm 9 \%$ ) could be improved by intensifying the therapy

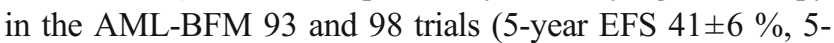
year OS $49 \pm 6 \%$ ) [2]. However, despite recent advances in the treatment, the optimal treatment strategy remains controversially discussed. Whereas some study groups treat nonDS-AMKL as very high risk, recommending allogeneic hematopoietic stem cell transplantation (HSCT) during first complete remission (CR1) [5], other study groups obtained superior survival rates with intensive chemotherapy alone, which were not further increased by allogeneic HSCT [8].

Similarly, the prognostic impact of cytogenetically defined subgroups could not be clearly defined, mostly due to the small size of the study cohorts. A limited number of specific genetic abnormalities characterizes non-DS-AMKL. Translocation $t(1 ; 22)$, which creates the RBM15-MKL1 (alias: OTT1/MAL) fusion protein, is unique to pediatric AMKL $[9,10]$. While the St. Jude AML 02 study proposed $t(1 ; 22)$ $(n=5)$ as a good prognostic marker [6], other studies suggested the opposite [9]. Also, the prognostic value of other frequently observed cytogenetic aberrations-such as complex karyotype, trisomy 8,19 , or 21, MLL-rearrangements, loss of chromosome 7 or 7q-, or der(3q) - remains open. Here, we report the clinical, cytogenetic, and therapeutic data of 97 children with non-DS-AMKL, treated uniformly according to the two sequential prospective multicenter studies AML-BFM 98 and AML-BFM 04.

\section{Patients and methods}

\section{Patients}

The AML-BFM 98 and AML-BFM 04 studies were randomized, controlled phase III studies running in 75 centers in Germany, Austria, Switzerland, and the Czech Republic. The AML-BFM 98 study opened in July 1, 1998 and closed in June 30, 2003. Between July 1, 2003 and April 30, 2004, the AML-BFM 98 Interim Study, continuing the best arm of the AML-BFM 98 trial, recruited further patients. The AML-BFM 04 study opened in April 2004 and closed in February 2014. The final protocols were approved by the protocol review committee of the German Cancer Aid (DKH) and by the ethics committee of the University of Münster.

Simultaneously, Germany, Austria, and Switzerland (BFM-Core group) participated in the prospective study on allogeneic HSCT vs. chemotherapy for high-risk (HR) childhood AML in first complete remission (AML CR1 HLA id) on behalf of the European Bone Marrow Transplantation (EBMT) Pediatric Working Party and the International BFM Study Group (I-BFMSG), which was approved by the ethics committee of the University of Tübingen. HR patients and family members were required to undergo HLA typing after the assignment to the risk group. All HR patients with a matched sibling donor were eligible for allogeneic HSCT in first complete remission.

The French-American-British (FAB) classification was used for the initial diagnosis of AML [1]. The diagnoses of the FAB M0 and M7 subtypes required confirmation by immunologic methods $[1,11]$. To characterize childhood acute megakaryoblastic leukemia, we reviewed 97 patients, diagnosed with AMKL by the BFM Study Group in the studies AML-BFM $98(n=37)$ and AML-BFM $04(n=60)$, including patients younger than 18 years with de novo AMKL. Patients with DS, myelosarcoma, secondary AML, or pretreatment of more than 2 weeks were excluded.

\section{Treatment plan}

Children with non-DS-AMKL were treated according to regimes AML-BFM 98 and AML-BFM 04, being similar for most parts (Fig. 1).

In AML-BFM 98, patients received chemotherapy with an induction of cytarabine (Ara-C), idarubicin, and etoposide (AIE) and thereafter HAM (high-dose cytarabine, mitoxantrone, cytarabine i.th.), followed by a randomized consolidation therapy (i.e., AI [cytarabine, idarubicin, cytarabine i.th.] and haM [high-dose cytarabine, mitoxantrone, cytarabine i.th.] or the BFM-type 6-week consolidation [6-thioguanine, prednisone, vincristine, idarubicin, cytarabine, cyclophosphamide, cytarabine i.th.]).

In AML-BFM 04, patients received a randomized induction therapy of cytarabine (Ara-C), liposomal daunorubicin (L-DNR), and etoposide (ADxE) or AIE [12]. After the second induction with HAM, consolidation therapy was randomized by introducing 2-CDA (2-chloro-2-deoxyadenosine) to AI as intensification [12-14].

Allogeneic HSCT should be performed in CR1 after consolidation according to the AML CR1 HLA id protocol. Children who were not transplanted in CR1 received one course of HAE as an intensification therapy (high-dose cytarabine and etoposide) and 1-year maintenance therapy (12 months; thioguanine, cytarabine, cytarabine i.th.). After 2006, the indication for allogeneic HSCT was restricted to patients with BM blasts $>5 \%$ after second induction. Allogeneic HSCT was performed in $23 \mathrm{SCT}$ centers as reported previously [15]. The recommended standard conditioning regimen for allogeneic HSCT in first complete remission was busulfan and cyclophosphamide. 


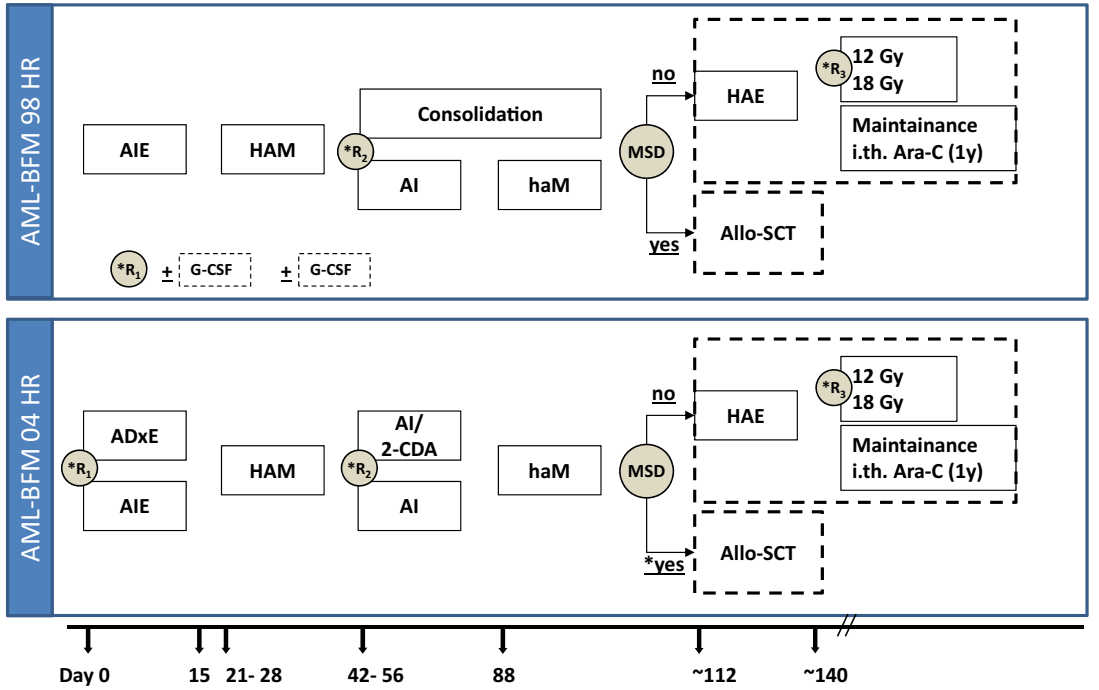

Fig. 1 Treatment schedule of AML-BFM 98 and AML-BFM 04 studies for high-risk patients. $A I E$ cytarabine/idarubicin/etoposide; $A D x E$ cytarabine/l-daunorubicin/etoposide; HAM high-dose cytarabine (3 g/ $\mathrm{m} 2 \mathrm{q} 12 \mathrm{~h}$ over 3 days)/mitoxantrone; $A I$ cytarabine/idarubicin; $A I / 2-$ $C D A$ cytarabine/idarubicin/2-chloro-2-deoxyadenosine; haM high-dose cytarabine (1 g/m2 q12h over 3 days)/mitoxantrone; consolidation 6-

\section{Cytogenetic and molecular genetic analyses}

Cytogenetic analyses were carried out and centrally reviewed in the AML-BFM reference laboratory in Giessen (AMLBFM 98) and Hannover (AML-BFM 04), Germany, as previously described [16]. Comprehensive cytogenetic data from $80 \%(n=78)$ of the included patients $(n=97)$ were available. Complete karyotypes were described according to the International System of Human Cytogenetic Nomenclature [17]. In the AML-BFM 04 study, sequencing of GATA1 was prospectively performed as previously described [18]. For 15 patients diagnosed before 2004, material was available for retrospective sequencing analysis.

\section{Statistics}

Complete remission (CR) was defined by fulfillment of the Cancer and Leukemia Group B (CALGB) criteria [19], early death (ED) being death before or within the first 6 weeks of treatment. Event-free survival (EFS) was defined as time from diagnosis to the first event. Events were death from any cause, early death, failure to achieve remission, relapse, and secondary malignancy. Failure to achieve remission was considered as an event on day 0 . Survival was defined as the time of diagnosis to death from any cause.

The Kaplan-Meier method was used to estimate survival rates [20]. Differences were compared with the 2-sided logrank test [21]. Standard errors (SEs) were obtained using Greenwoods formula. Cumulative incidence of relapse and death in CR were calculated by the method of Kalbfleisch thioguanine/prednisone/vincristine/idarubicin/cytarabine/cyclophosphamide; HAE high-dose cytarabine ( $3 \mathrm{~g} / \mathrm{m} 2 \mathrm{q} 12 \mathrm{~h}$ over 3 days)/ etoposide; MSD matched sibling donor; asterisk indicates until 2006; CNS irradiation; maintenance 12 months thioguanine/cytarabine; RI first random assignment; $R 2$ second random assignment; $R 3$ third random assignment

and Prentice and compared with Gray's test. The Cox proportional hazards model has been used to obtain the estimates and the $95 \%$ confidence interval of the relative risk for prognostic factors [22]. Differences in the distribution of individual parameters among patient subsets were analyzed using the chisquared test or Fisher's exact test for categorized variables and the Mann-Whitney $U$ test for continuous variables. The effect of HSCT on survival was tested using the Mantel-Byar method for comparisons of patients treated or not treated with HSCT. For graphic presentation, patients without HSCT and EFS below the median time to transplantation ( 0.4 years $)$ were excluded. Follow-up was as of June 2014. Computations were performed using SAS (Statistical Analysis System Version 9.3; SAS Institute, Cary, NC).

\section{Results}

\section{Patient characteristics}

Of 1316 pediatric patients with AML enrolled in the population-based studies AML-BFM 98 and AML-BFM 04, $97(7.4 \%)$ presented with de novo non-DS-AMKL. Patient characteristics are summarized in Table 1 . The median age at diagnosis was 1.44 years (range $0-15$ years), being notably younger than for other AML subtypes (9.98 years; $P_{\text {Fisher's }}<$ $0.0001)$. The mean white blood cell (WBC) count of children with de novo AMKL $\left(16.5 \times 10^{9} / \mathrm{L}\right)$ was lower than of children with other AML subtypes $\left(58.5 \times 10^{9} / \mathrm{L}\right)$. Additionally, initial CNS involvement occurred more frequently among other 
Table 1 Patient characteristics

\begin{tabular}{|c|c|c|c|c|c|c|c|c|c|c|}
\hline & \multicolumn{7}{|c|}{ AMKL (FAB M7) } & \multirow{2}{*}{\multicolumn{3}{|c|}{$\frac{\text { Other AML subtypes }}{\text { AML-BFM 98+04 }}$}} \\
\hline & \multicolumn{2}{|c|}{ Total } & \multicolumn{2}{|c|}{ AML-BFM 98} & \multicolumn{3}{|c|}{ AML-BFM 04} & & & \\
\hline & $N$ & $(\%)$ & $N$ & $(\%)$ & $N$ & $(\%)$ & $P^{\mathrm{a}}$ & $N$ & $(\%)$ & $P^{\mathrm{b}}$ \\
\hline \multicolumn{11}{|l|}{ Gender } \\
\hline Male & 53 & 54.6 & 21 & 56.8 & 32 & 53.3 & & 625 & 51.3 & \\
\hline Female & 44 & 45.4 & 16 & 43.2 & 28 & 46.7 & 0.83 & 594 & 48.7 & 0.52 \\
\hline \multicolumn{11}{|l|}{ Age, years } \\
\hline$<2$ & 64 & 66.0 & 24 & 64.9 & 40 & 66.7 & & 241 & 19.8 & \\
\hline $2-5$ & 23 & 23.7 & 6 & 16.2 & 17 & 28.3 & & 170 & 13.9 & \\
\hline$\geq 6$ & 10 & 10.3 & 7 & 18.9 & 3 & 5.0 & 0.06 & 808 & 66.3 & $<0.001$ \\
\hline \multicolumn{11}{|l|}{$\mathrm{WBC}, 10^{9} / \mathrm{L}$} \\
\hline$<20$ & 75 & 77.3 & 24 & 64.9 & 51 & 85.0 & & 631 & 51.8 & \\
\hline$>20$ & 22 & 22.7 & 13 & 35.1 & 9 & 15.0 & 0.03 & 587 & 48.2 & $<0.001$ \\
\hline \multicolumn{11}{|l|}{ CNS involvement } \\
\hline No & 92 & 94.8 & 35 & 100.0 & 57 & 96.6 & & 1036 & 87.6 & \\
\hline Yes & 2 & 2.1 & - & - & 2 & 3.4 & 0.53 & 147 & 12.4 & $<0.001$ \\
\hline \multicolumn{11}{|l|}{ BM day $15+28$} \\
\hline$\leq 5 \%$ of blasts & 74 & 77.9 & 24 & 66.7 & 50 & 84.7 & & 815 & 71.1 & \\
\hline$>5 \%$ of blasts & 21 & 21.1 & 12 & 33.3 & 9 & 15.3 & 0.05 & 331 & 28.9 & 0.16 \\
\hline \multicolumn{11}{|l|}{ Anamnesis } \\
\hline$<3$ weeks & 38 & 41.8 & 16 & 43.2 & 22 & 36.7 & & 680 & 57.2 & \\
\hline$\geq 3$ weeks & 53 & 58.2 & 18 & 48.6 & 35 & 48.3 & 0.51 & 508 & 42.8 & 0.004 \\
\hline \multicolumn{11}{|l|}{ Allo HSCT in 1.CR } \\
\hline Yes & 18 & 22.8 & 9 & 27.3 & 9 & 18.8 & & 113 & 89.5 & \\
\hline No & 63 & 77.8 & 24 & 72.7 & 39 & 81.3 & 0.35 & 962 & 10.5 & $<0.001$ \\
\hline
\end{tabular}

${ }^{a}$ AML-BFM 98 vs. AML-BFM 04

${ }^{\mathrm{b}}$ AMKL vs. other AML subtypes

AML subtypes (12.4\%) than among AMKL patients $(2.1 \%)$. Patients with AMKL frequently presented with a longer disease history. Only $41.8 \%$ of AMKL patients had an anamnesis less than 3 weeks, as compared to $57.2 \%$ of children with other AML subtypes.

Comparing AML-BFM 98 and AML-BFM 04, the study population did not significantly differ. We only noted statistical significance in the mean $\mathrm{WBC}$ of $19.7 \times 10^{9} / \mathrm{L}$ vs. $14.5 \times$ $10^{9} / \mathrm{L}(P=0.002)$.

\section{Treatment outcome}

With a 5-year OS of $60 \pm 5 \%$ (Fig. 2) and 5-year EFS of $47 \pm$ $5 \%$, children with AMKL revealed a significantly poorer outcome as compared to the total group of children with other AML subtypes (Fig. 2; 5-year EFS 52 $\pm 1 \%, P_{\text {log rank }}=0.079$ ). The complete remission (CR) rate of non-DS-AMKL patients was comparable to other children with HR-AML (defined by the reported criteria of the AML-BFM study group [14, 23]). Eighty-one patients $(83.6 \%)$ achieved CR, $32 \%(n=32)$ relapsed after first CR, and $4.1 \%(n=4)$ died in first CR. Thirteen patients $(13.4 \%)$ were partial- and non-responders (PR; NR) to induction therapy and 3.1\% $(n=3)$ suffered from early death (ED). The cumulative incidence of relapse (CIR)

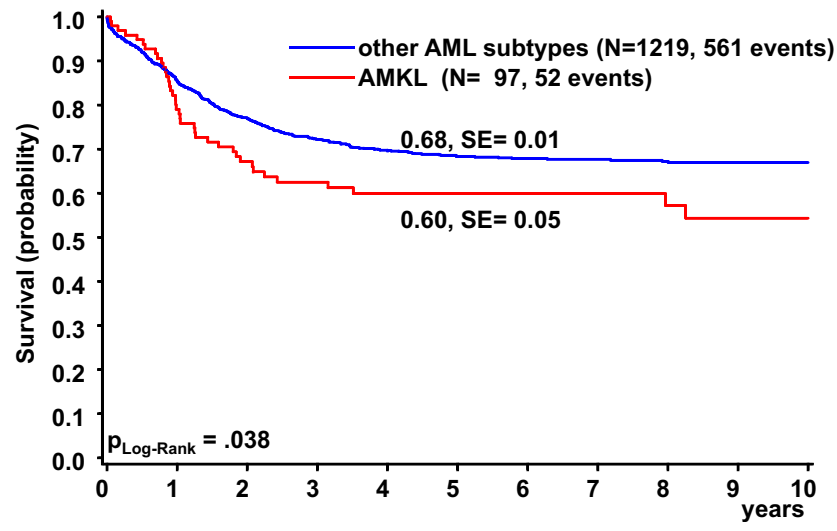

Fig. 2 Overall survival of patients diagnosed with non-DS de novo AMKL $(n=97)$ or other AML subtypes $(n=1219)$ in the AML-BFM 98 and AML-BFM 04 studies. Five-year OS is given 
was $45 \pm 5 \%$ compared to $39 \pm 1 \%\left(P_{\text {Gray }}=0.07\right)$ for patients with other AML subtypes.

Comparing AML-BFM 98 to AML-BFM 04 (Fig. 1), the 5-year EFS increased and the 5-year OS of AMKL patients increased significantly from $45 \pm 8 \%$ to $70 \pm 6 \%$ (Fig. $3 a-b$ ), while CIR decreased from $46 \pm 8 \%$ to $23 \pm 6 \%$ (Fig. $3 \mathrm{c}$ ).

Although the introduction of liposomal daunorubicin (LDNR or Dx) in the induction of AML-BFM 04 (randomization of ADxE vs. AIE) resulted in no significant improvement

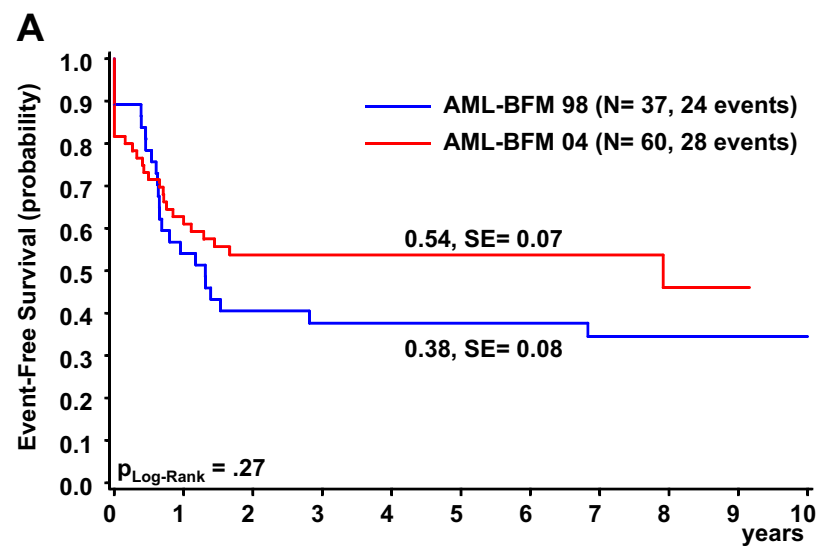

B
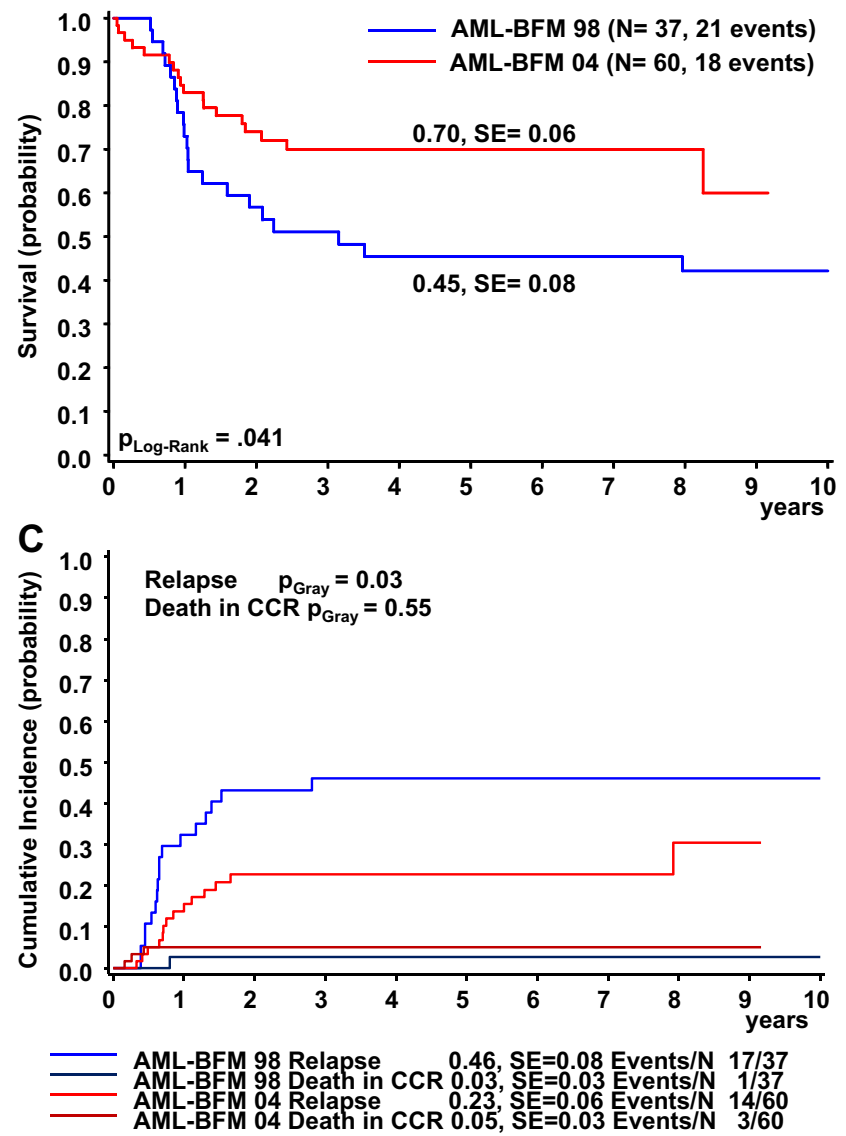

Fig. 3 Outcome of AMKL patients in the AML-BFM 98 or AML-BFM 04 studies: a Event-free survival. b Overall survival. c Cumulative incidence of relapse/death. Five-year probabilities are given regarding 5-year EFS and 5-year OS (56 $\pm 12 \%$ vs. $52 \pm 8 \%$, $P_{\log \text { rank }}=0.84 ; 74 \pm 11 \%$ vs. $\left.68 \pm 8 \%, P_{\log \text { rank }}=0.57\right)$, only $15.3 \%$ of AMKL patients in AML-BFM 04 (ADxE and AIE) displayed a poor treatment response, as assessed by bone marrow morphology on day 15 and day 28 of therapy ( $>5 \%$ blast cells). This is a clear improvement to AML-BFM 98 with $33.3 \%$ (Table 1). In both cohorts together (AML-BFM 98 and 04), those poor responders had an estimated 5-year OS of only $35 \pm 11 \%$ and 5-year EFS of $12 \pm 8 \%$, while good responders ( $\leq 5 \%$ BM blasts on day 15 and day 28$)$ showed a 5-year OS of $66 \pm 6 \%\left(P_{\log \text { rank }}=0.021\right)$ and 5-year EFS of $56 \pm 6 \%\left(P_{\log \text { rank }}=0.0001\right)$ (Fig. $\left.4 \mathrm{a}, \mathrm{b}\right)$. The introduction of $2-$ CDA to the consolidation therapy did not have an impact on the survival rates on AMKL patients.

\section{Postremission management}

Until 2006, allogeneic HSCT in CR1 was recommended for all patients with an HLA compatible sibling donor. To assess the therapeutic effect of allogeneic HSCT compared to chemotherapy alone, we performed as-treated survival analyses taking time to transplant into account. For graphical display patients who did not receive HSCT and had an event before
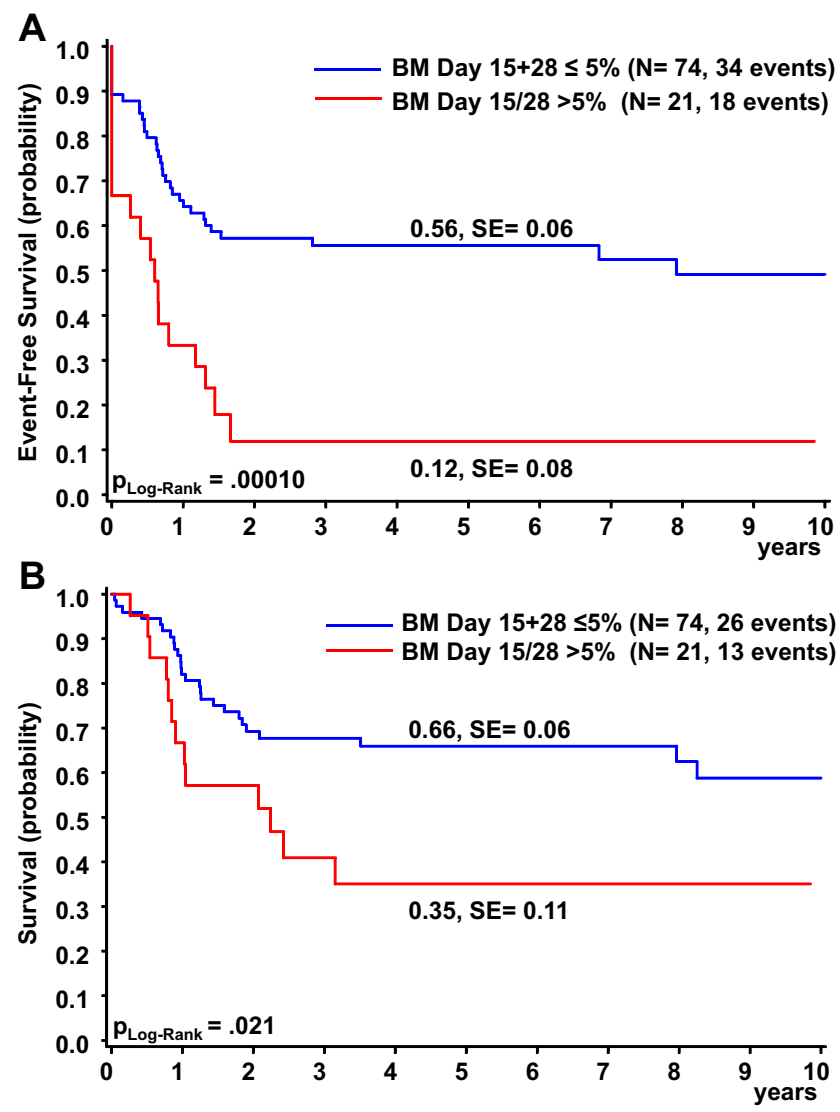

Fig. 4 Outcome of AMKL patients based on treatment response: a Event-free survival. b Overall survival. c Cumulative incidence of relapse/death. Five-year probabilities are given. Treatment response was evaluated by bone marrow morphology ( $>5 \%$ blasts on day 15 or 28 ) 
the median time until transplantation ( 0.4 years) were excluded. In AML-BFM 98, allogeneic HSCT in CR1 was performed on 9 (27\%) non-DS-AMKL patients, whereas 24 patients $(73 \%)$ were treated by chemotherapy alone. In AML-BFM 04, the percentages were 19 and $81 \%$. The outcome was not significantly different in patients undergoing allogeneic HSCT rather than chemotherapy alone in AMLBFM 98 (5-year OS: $56 \pm 17 \%$ vs. $49 \pm 11 \%, P_{\text {Mantel-Byar }}=$ 0.95 ; 5 -year EFS $44 \pm 17 \%$ vs. $45 \pm 11 \%, P_{\text {Mantel-Byar }}=0.6$ ) and AML-BFM 04 (5-year OS: $88 \pm 12 \%$ vs. $72 \pm 8 \%, P_{\text {Mantel- }}$ Byar $=0.83$; 5 -year EFS: $88 \pm 12 \%$ vs. $65 \pm 8 \%, P_{\text {Mantel-Byar }}=$ 0.58 )(Supplementary Fig. S1). Likewise, we also observed no benefit from allogeneic HSCT when analyzing the total cohort of AMKL patients (AML-BFM 98 and 04; Fig. 5a, b, Table 2).

\section{Cytogenetic analyses}

Cytogenetic analyses were performed for $78(80.4 \%)$ children with non-DS-AMKL (Tables 1 and 2). Of note, neither complex karyotype (three or more independent abnormalities
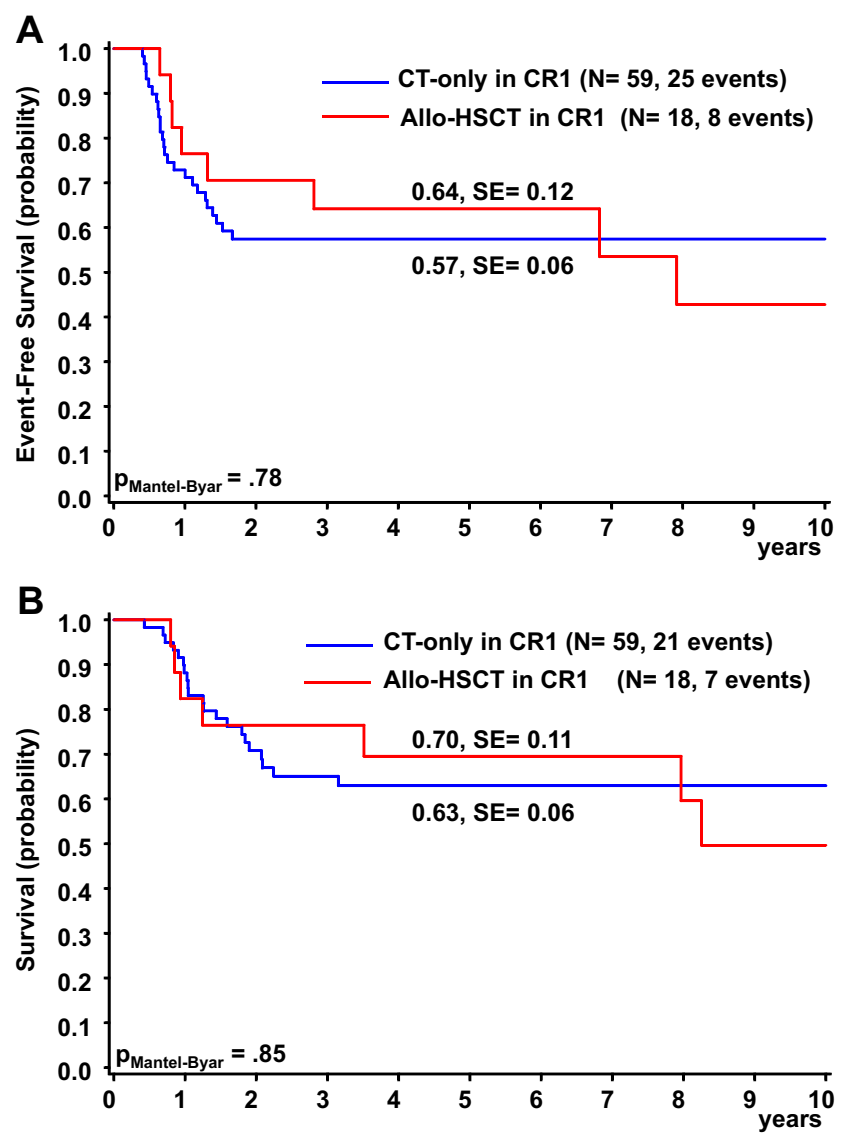

Fig. 5 Outcome of AMKL patients assigned to allogeneic HSCT in 1.CR or to chemotherapy only: a Event-free survival. b Overall survival. Fiveyear probabilities are given. Patients who did not receive HSCT and had an event before the median time until transplantation ( 0.4 years) were excluded including at least one structural abnormality), MLL rearrangement nor monosomy $7 / 7 \mathrm{q}$ - was associated with a significantly worse outcome compared to patients without these aberrations (Table 2, Supplementary Fig. S2A-C). The translocation $t(1 ; 22)(\mathrm{p} 13 ; \mathrm{q} 13)$ was found in 8 patients $(10.3 \%$; patient characteristics summarized in Supplementary Table S1), who had a significantly worse 5 -year EFS $(38 \pm 17 \%)$ than patients without this translocation $\left(53 \pm 6 \%, P_{\log \text { rank }}=0.039\right.$; Table 2 and Supplementary Fig. S2D-F).

Other recurrent aberrations were mainly numerical changes, such as gain of chromosome 8 or 21. Gain of chromosome 21 was associated with better survival rates (5-year OS $=80 \pm$ $9 \%$ vs. $54 \pm 7 \%, P_{\log \text { rank }}=0.034$; Supplementary Fig. S2G-I).

\section{GATA1-status}

Sequencing of the hematopoietic transcription factor GATA1 was performed on 53 patients. None of these children was previously diagnosed with Down syndrome (constitutional trisomy 21 or trisomy 21 mosaic) or had a history of transient abnormal myelopoiesis (TAM) during the neonatal period. Six patients $(11.3 \%)$ were positive for a GATA1-mutation, and a gain of chromosome 21 in the leukemic blasts was detected (Table 3). Based on these findings, four patients were diagnosed with trisomy 21 mosaic and two with an acquired trisomy 21. Interestingly, the leukemic blasts of all six patients showed CD7 surface marker expression (Table 3), a common feature of DS-AMKL [24]. Two patients with trisomy 21 mosaic were treated by the reduced intensity ML-DS 2006 treatment recommendations and are in continuous CR without additional therapy. From the remaining four patients, who were treated by standard AML-BFM protocols, one child received matched sibling donor (MSD) HSCT after NR and another one received HSCT after CR2 from a mismatched unrelated donor. Each child with GATAl-mutation maintains in continuous CR (Table 3).

\section{Multivariate analysis}

In the multivariate analysis for 5-year EFS, including sex, age, WBC more than $20 \times 10^{9} / \mathrm{L}$, BM blasts more than $5 \%$ on day 15 or 28 , as well as cytogenetic subgroups (normal and complex karyotype, $t(1 ; 22)(\mathrm{p} 13 ; \mathrm{q} 13)$, gain of chromosome $8(+8)$, gain of chromosome $21(+21)$, monosomy 7 and $\operatorname{der}(3))$ as risk factors, only poor treatment response ( $>5 \%$ blasts after 15 or 28 days; $\mathrm{RR}=4.39 ; 95 \% \mathrm{CI}, 1.97-9.78 ; P_{x 2}=0.0003$ ) was of independent prognostic significance (Table 4). For translocation $t(1 ; 22)(\mathrm{p} 13 ; \mathrm{q} 13)$, which was statistically significant in the univariate analysis, multivariate analysis indicated a trend towards poor prognosis $\left(P_{x 2}=0.07\right.$; Table 4$)$. 
Table 2 5-year EFS/OS of defined subgroups in AMKL (AML-BFM 98+04)

\begin{tabular}{|c|c|c|c|c|c|c|c|}
\hline$(n=97)$ & $N$ & Events & EFS (\%) & $P$ & Deaths & OS $(\%)$ & $P$ \\
\hline \multicolumn{8}{|l|}{ Gender } \\
\hline Male & 53 & 28 & $47 \pm 8$ & & 21 & $60 \pm 7$ & \\
\hline Female & 44 & 24 & $47 \pm 7$ & 0.95 & 18 & $60 \pm 8$ & 0.92 \\
\hline \multicolumn{8}{|l|}{ Age, years } \\
\hline$<2$ & 64 & 35 & $46 \pm 6$ & & 25 & $60 \pm 6$ & \\
\hline $2-9$ & 23 & 11 & $50 \pm 11$ & & 8 & $64 \pm 10$ & \\
\hline$\geq 10$ & 10 & 6 & $50 \pm 16$ & 0.93 & 6 & $48 \pm 16$ & 0.36 \\
\hline \multicolumn{8}{|l|}{ WBC, $10^{9} / \mathrm{L}$} \\
\hline$<20$ & 75 & 41 & $46 \pm 6$ & & 31 & $59 \pm 6$ & \\
\hline$\geq 20$ & 22 & 11 & $52 \pm 11$ & 0.87 & 8 & $65 \pm 11$ & 0.99 \\
\hline \multicolumn{8}{|l|}{ BM day $15+28$} \\
\hline$\leq 5 \%$ of blasts & 74 & 34 & $56 \pm 6$ & & 26 & $66 \pm 6$ & \\
\hline$>5 \%$ of blasts & 21 & 18 & $12 \pm 8$ & $<0.001$ & 13 & $35 \pm 11$ & 0.02 \\
\hline \multicolumn{8}{|l|}{ Anamnesis } \\
\hline$<3$ weeks & 38 & 15 & $64 \pm 8$ & & 13 & $69 \pm 8$ & \\
\hline$\geq 3$ weeks & 53 & 36 & $31 \pm 7$ & 0.01 & 25 & $51 \pm 7$ & 0.23 \\
\hline \multicolumn{8}{|l|}{ Postremission management } \\
\hline Allogeneic HSCT in 1.CR & 18 & 8 & $64 \pm 12$ & & 7 & $70 \pm 11$ & \\
\hline Chemotherapy only & 59 & 25 & $57 \pm 6$ & 0.78 & 21 & $63 \pm 6$ & 0.85 \\
\hline \multicolumn{8}{|l|}{ Cytogenetics } \\
\hline Normal & 13 & 5 & $62 \pm 13$ & 0.30 & 5 & $60 \pm 14$ & 0.86 \\
\hline Complex & 25 & 11 & $55 \pm 10$ & 0.60 & 7 & $71 \pm 9$ & 0.17 \\
\hline$t(1 ; 22)(\mathrm{p} 13 ; \mathrm{q} 13)$ & 8 & 6 & $38 \pm 17$ & 0.04 & 4 & $63 \pm 17$ & 0.30 \\
\hline 11q23-aberrations & 9 & 4 & $51 \pm 18$ & 0.93 & 3 & $63 \pm 17$ & 0.89 \\
\hline $\operatorname{der}(3)$ & 3 & 1 & $67 \pm 27$ & 0.50 & 1 & $67 \pm 27$ & 0.82 \\
\hline+21 & 21 & 8 & $60 \pm 11$ & 0.23 & 4 & $80 \pm 9$ & 0.03 \\
\hline+8 & 16 & 8 & $56 \pm 12$ & 0.74 & 6 & $68 \pm 12$ & 0.62 \\
\hline Monosomy 7 & 3 & 2 & $33 \pm 27$ & 0.57 & 2 & $33 \pm 27$ & 0.19 \\
\hline Hyperdiploid & 3 & 2 & $33 \pm 27$ & 0.46 & 2 & $33 \pm 27$ & 0.14 \\
\hline
\end{tabular}

\section{Discussion}

The overall outcome of AMKL (excluding DS) was extremely poor in the early AML-BFM 87 trial (EFS $11 \pm 7 \%, 5$-year OS $21 \pm 9 \%$ ), which could be significantly improved in the following trials AML-BFM 93 and 98 (5-year EFS $41 \pm 6 \%$, 5year OS $49 \pm 6 \%$ ) [2] by introducing idarubicin as well as high-dose cytarabine plus mitoxantrone to the protocol. With a 5 -year OS of $70 \pm 6 \%$ in AML-BFM 04, we now achieved the highest survival rate for pediatric non-DS-AMKL patients within the BFM studies.

Our study with 97 patients demonstrates that AMKL is nowadays not necessarily associated with a poor outcome, as we and others previously reported (Athale 2001: SJCRH, $n=28$, 2-year EFS/OS 14/14 \%; O'Brien 2013: AML02 ( $n=$ 26), 3-year EFS/OS 49/54 \%; POG 9421 ( $n=49), 5$-year EFS/ OS 35/36 \%; Barnard 2007: CCG $2891(n=53), 5$-year EFS/ OS 23/28\%) [5-7]. Although we confirmed that AMKL constitutes an AML subgroup with inferior outcome compared to the whole group of AML patients, we could achieve a steep increase in the survival rates by continuous development of the AML-BFM therapy protocols. These results support a Japanese study on a cohort of 21 patients with AMKL, reporting 10-year EFS and OS of 57 and $76 \%$, respectively [8]. Hence, intensification of the already very intensive therapy regimen and better supportive care may result in improved prognosis in AMKL patients.

Interestingly, the improved survival rates of the AMLBFM 04 study could not be attributed to advances in allogeneic HSCT. Thus, we could not confirm previous studies suggesting that survival rates are significantly better after allogeneic HSCT. The St Jude Children's Research Hospital reported 2-year OS (30\%) after allogeneic HSCT compared to $0 \%$ after chemotherapy alone, stating that allogeneic HSCT during remission offers the best chance of cure [5]. The European Group for Blood and Marrow Transplantation described that the 3-year OS was $82 \%$ in children after allogeneic HSCT. In their study, also DS-AMKL patients were included, which 


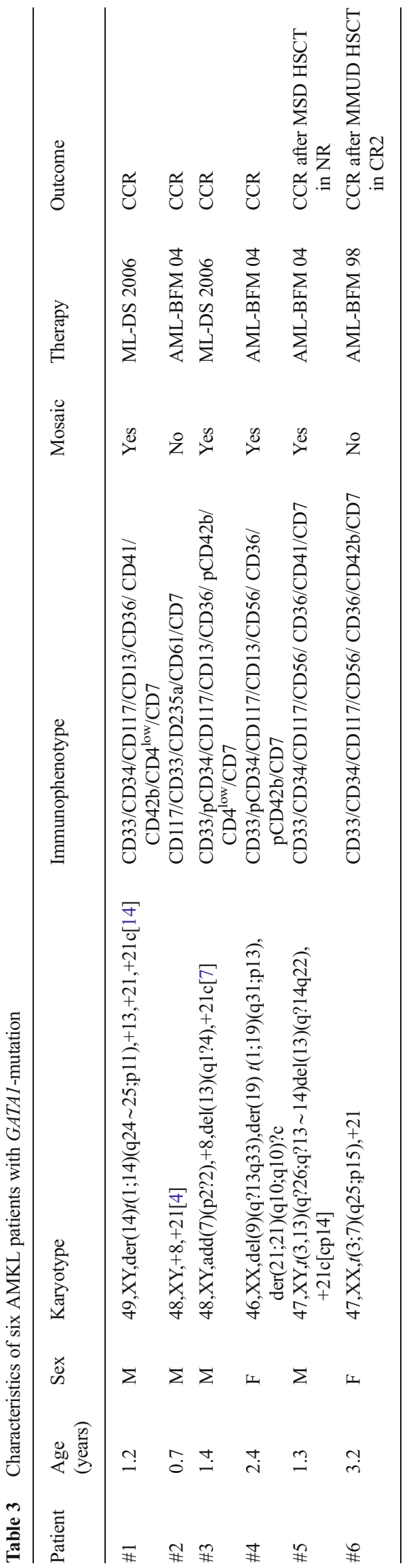

Table 4 Multivariable Cox regression analysis of clinical factors and cytogenetics for EFS

\begin{tabular}{llll}
\hline & RR & $95 \% \mathrm{CI}$ & $P$ \\
\hline Gender & 1.0 & $0.5-2.1$ & 0.96 \\
Age & 1.1 & $0.6-1.9$ & 0.81 \\
WBC $>20 \times 109 / \mathrm{L}$ & 0.9 & $0.4-2.3$ & 0.90 \\
BM day $15 / 25,>5 \%$ blasts & 4.4 & $2.0-9.8$ & 0.0003 \\
Cytogenetics & & & \\
$\quad$ Normal & 0.6 & $0.2-2.1$ & 0.47 \\
Complex & 1.1 & $0.4-2.9$ & 0.84 \\
$t(1 ; 22)(\mathrm{p} 13 ; \mathrm{q} 13)$ & 3.9 & $0.9-16.3$ & 0.07 \\
$\quad+8$ & 0.7 & $0.2-1.8$ & 0.43 \\
+21 & 0.7 & $0.3-1.9$ & 0.50 \\
Monosomy 7 & 0.4 & $0.1-2.5$ & 0.35 \\
der(3) & 0.5 & $0.0-4.5$ & 0.51 \\
\hline
\end{tabular}

confused the interpretation of the results [25]. In accordance to Hama et al. [8], the survival rates between patients, who were transplanted in CR1 or who received chemotherapy alone, did not differ significantly, resulting in no proven benefit of allogeneic HSCT in CR1 in our series for non-DSAMKL. Still, because of the small number of AMKL patients with allogeneic HSCT in CR1, the results should be interpreted carefully.

The response to induction therapy is of independent (multivariate analysis) and strong prognostic relevance. Patients with more than $5 \%$ bone marrow blasts on day 15 or 28 of therapy have a poor 5 -year OS of only $35 \pm 11 \%$. Still, the number of non-responders could be reduced in AML-BFM 04 (8\%), compared to AML-BFM 98 (17.1\%). PCR- or flow cytometry-based residual disease monitoring may help to distinguish between increased numbers of normal myeloblasts in the regenerating bone marrow after chemotherapy and persistence of malignant blasts to more accurately determine the treatment response in the future [26-28].

Our cytogenetic analyses mark a difference to earlier reports. Carroll et al. [9] and Duchayne et al. [10] described the translocation $t(1 ; 22)(\mathrm{p} 13 ; \mathrm{q} 13)$ to be a good prognostic factor. Contrarily, we demonstrated for 8 patients $(10.1 \%$ of our cohort) a significantly lower 5-year EFS compared to patients without this translocation. Though this may be attributable to the small number of patients and the high NR rate, our report puts a clear role of $t(1 ; 22)$ as a good prognostic indicator into question. Interestingly, neither monosomy 7/7q-, complex karyotype nor 11q23 aberrations indicated a poor prognosis. A good prognostic factor in our study was gain of chromosome 21, which we found in $21.9 \%$ of the patients (Supplementary Fig. 2G-I). This result is represented in children, who carried a GATA1-mutation $(n=6)$. Based on the GATA1-status, which is strongly associated with DS-AMKL, four of these patients were diagnosed with trisomy 21-mosaicism that was 
previously unknown. Two patients did not have constitutional trisomy 21 , suggesting that trisomy 21 was acquired as an early step during leukemogenesis. All six patients are in continuous remission after treatment, indicating a similar biology and treatment response of AMKL with acquired or constitutional (DS-AMKL) trisomy 21 in conjunction with GATA1s. Therefore, it is crucial to perform GATA1 mutation analysis on each patient with pediatric AMKL and it may not be advisable that children with GATA1-mutations are stratified to the high-risk arm of AML protocols like other AMKL patients. All children with GATA1s-mutation showed CD7 surface marker expression, which could serve as a marker to predict the cases with GATA1s-mutation [24]. Based on previous experience [29], two patients of our cohort with trisomy 21 mosaic and GATA1 mutation were successfully treated with a reduced intensity regimen for DS-AMKL patients (according to treatment recommendation of the ML-DS 2006 registry). Similar to DSAMKL, reduced intensity regimens seem efficient in children with trisomy 21 mosaic and overtreatment should be avoided.

Thus, the focus on cytogenetic analyses remains of high prognostic importance. Especially, the role of the newly defined recurrent cytogenetically cryptic NUP98-JARID1A fusion $(t(11 ; 15)(\mathrm{p} 15 ; \mathrm{q} 35))$ and CBFA2T3-GLIS2-fusion (inv(16)) needs to be addressed [30, 31]. For this reason, international collaborative projects on pediatric non-DS-AMKL are essential to study a broad range of chromosomal abnormalities, which might improve statistical analyses and therefore define a better risk stratification [32]. Current efforts from the international BFM study group might address this issue.

In summary, improved intensified chemotherapy and experience of medical staff secure the step-wise and considerable increase of long-term survival for children with non-DS de novo AMKL. We could not prove a clear benefit for allogeneic HSCT in first complete remission for these patients.

Acknowledgments The authors thank all colleagues, data managers, and technicians of the participating hospitals for their valuable cooperation. A complete listing of the participating AML-BFM institutions and investigators appears in the Appendix. We thank JE Müller (Hannover) for competent data management.

Funding This work was supported by the Deutsche Krebshilfe e.V. J.H.K. is a fellow of the Emmy Noether Programme from the German Research Foundation (DFG; KL-2374/2-1).

Conflict of interest D.R. is a member of the advisory board from Galen. The remaining authors declare no competing financial interests.

Open Access This article is distributed under the terms of the Creative Commons Attribution 4.0 International License (http:// creativecommons.org/licenses/by/4.0/), which permits unrestricted use, distribution, and reproduction in any medium, provided you give appropriate credit to the original author(s) and the source, provide a link to the Creative Commons license, and indicate if changes were made.

\section{References}

1. Bennett JM, Catovsky D, Daniel MT, Flandrin G, Galton DA, Gralnick HR et al (1985) Criteria for the diagnosis of acute leukemia of megakaryocyte lineage (M7). A report of the French-AmericanBritish Cooperative Group. Ann Intern Med 103(3):460-462

2. Reinhardt D, Diekamp S, Langebrake C, Ritter J, Stary J, Dworzak $M$ et al (2005) Acute megakaryoblastic leukemia in children and adolescents, excluding Down's syndrome: improved outcome with intensified induction treatment. Leukemia 8:1495-1496

3. Creutzig U, Ritter J, Vormoor J, Ludwig WD, Niemeyer C, Reinisch I et al (1996) Myelodysplasia and acute myelogenous leukemia in Down's syndrome. A report of 40 children of the AML-BFM Study Group. Leukemia 10(11):1677-1686

4. Zipursky A, Peeters M, Poon A (1987) Megakaryoblastic leukemia and Down's syndrome: a review. Pediatr Hematol Oncol 4(3):211-230

5. Athale UH, Razzouk BI, Raimondi SC, Tong X, Behm FG, Head DR et al (2001) Biology and outcome of childhood acute megakaryoblastic leukemia: a single institution's experience. Blood 97(12):3727-3732

6. O'Brien MM, Cao X, Pounds S, Dahl GV, Raimondi SC, Lacayo NJ et al (2013) Prognostic features in acute megakaryoblastic leukemia in children without Down syndrome: a report from the AML02 multicenter trial and the Children's Oncology Group Study POG 9421. Leukemia 27(3):731-734

7. Barnard DR, Alonzo TA, Gerbing RB, Lange B, Woods WG (2007) Children's Oncology Group. Comparison of childhood myelodysplastic syndrome, AML FAB M6 or M7, CCG 2891: report from the Children's Oncology Group. Pediatr Blood Cancer 49(1):17-22

8. Hama A, Yagasaki H, Takahashi Y, Nishio N, Muramatsu H, Yoshida N et al (2008) Acute megakaryoblastic leukaemia (AMKL) in children: a comparison of AMKL with and without Down syndrome. Br J Haematol 140(5):552-561

9. Carroll A, Civin C, Schneider N, Dahl G, Pappo A, Bowman P et al (1991) The $t(1 ; 22)(p 13 ; q 13)$ is nonrandom and restricted to infants with acute megakaryoblastic leukemia: a Pediatric Oncology Group Study. Blood 78(3):748-752

10. Duchayne E, Fenneteau O, Pages MP, Sainty D, Arnoulet C, Dastugue N et al (2003) Acute megakaryoblastic leukaemia: a national clinical and biological study of 53 adult and childhood cases by the Groupe Francais d'Hematologie Cellulaire (GFHC). Leuk Lymphoma 44(1):49-58

11. Bennett JM, Catovsky D, Daniel MT, Flandrin G, Galton DA, Gralnick HR et al (1991) Proposal for the recognition of minimally differentiated acute myeloid leukaemia (AML-MO). Br J Haematol 78(3):325-329

12. Creutzig U, Zimmermann M, Dworzak MN, Ritter J, Schellong G, Reinhardt D (2013) Development of a curative treatment within the AML-BFM studies. Klin Padiatr 225(Suppl 1):S79-S86

13. Creutzig U, Zimmermann M, Bourquin JP, Dworzak MN, Fleischhack G, Graf N et al (2013) Randomized trial comparing liposomal daunorubicin with idarubicin as induction for pediatric acute myeloid leukemia: results from Study AML-BFM 2004. Blood 122(1):37-43

14. Creutzig U, Zimmermann M, Lehrnbecher T, Graf N, Hermann J, Niemeyer CM et al (2006) Less toxicity by optimizing chemotherapy, but not by addition of granulocyte colony-stimulating factor in children and adolescents with acute myeloid leukemia: results of AML-BFM 98. J Clin Oncol 24(0732-183; 27):4499-4506

15. Klusmann JH, Reinhardt D, Zimmermann M, Kremens B, Vormoor J, Dworzak M et al (2012) The role of matched sibling donor allogeneic stem cell transplantation in pediatric high-risk acute myeloid leukemia: results from the AML-BFM 98 study. Haematologica 97(1):21-29

16. von Neuhoff C, Reinhardt D, Sander A, Zimmermann M, Bradtke J, Betts DR et al (2010) Prognostic impact of specific chromosomal aberrations in a large group of pediatric patients with acute myeloid 
leukemia treated uniformly according to trial AML-BFM 98. J Clin Oncol 28(0732-183; 16):2682-2689

17. Mitelman F, Karger S (1995) An international system for human cytogenetic nomenclature

18. Alford KA, Reinhardt K, Garnett C, Norton A, Bohmer K, von Neuhoff $C$ et al (2011) Analysis of GATA1 mutations in Down syndrome transient myeloproliferative disorder and myeloid leukemia. Blood 118(8):2222-2238

19. Cheson BD, Cassileth PA, Head DR, Schiffer CA, Bennett JM, Bloomfield CD et al (1990) Report of the National Cancer Institute-sponsored workshop on definitions of diagnosis and response in acute myeloid leukemia. J Clin Oncol 8(5):813-819

20. Kaplan EL, Meier P (1958) Nonparametric estimation from incomplete observations. J Am Stat Assoc 53:457-481

21. Mantel N (1966) Evaluation of survival data and two rank order statistics arising in its consideration. Cancer Chemother Rep 50: $163-170$

22. Cox DR (1972) Regression models and life tables. J R Stat Soc 34: 187

23. Creutzig U, Zimmermann M, Bourquin JP, Dworzak MN, Fleischhack G, Graf N et al (2013) Randomized trial comparing liposomal daunorubicin with idarubicin as induction for pediatric acute myeloid leukemia: results from Study AML-BFM 2004. Blood 122(1):37-43

24. Langebrake C, Creutzig U, Reinhardt D (2005) Immunophenotype of Down syndrome acute myeloid leukemia and transient myeloproliferative disease differs significantly from other diseases with morphologically identical or similar blasts. Klin Padiatr 217(3): 126-134

25. Garderet L, Labopin M, Gorin NC, Polge E, Baruchel A, Meloni G et al (2005) Hematopoietic stem cell transplantation for de novo acute megakaryocytic leukemia in first complete remission: a retrospective study of the European Group for Blood and Marrow Transplantation (EBMT). Blood 105(1):405-409

26. Pine SR, Guo Q, Yin C, Jayabose S, Levendoglu-Tugal O, Ozkaynak MF et al (2005) GATA1 as a new target to detect minimal residual disease in both transient leukemia and megakaryoblastic leukemia of Down syndrome. Leuk Res 29(11): 1353-1356

27. Takeda A, Shimada A, Hamamoto K, Yoshino S, Nagai T, Fujii Y et al (2014) Detection of RBM15-MKL1 fusion was useful for diagnosis and monitoring of minimal residual disease in infant acute megakaryoblastic leukemia. Acta Med Okayama 68(2):119-123

28. Langebrake C, Creutzig U, Dworzak M, Hrusak O, Mejstrikova E, Griesinger $\mathrm{F}$ et al (2006) Residual disease monitoring in childhood acute myeloid leukemia by multiparameter flow cytometry: the MRD-AML-BFM Study Group. J Clin Oncol 24(22):3686-3692

29. Reinhardt D, Reinhardt K, Neuhoff C, Sander A, Klusmann JH, Pekrun A et al (2012) GATA1-mutation associated leukemia in children with trisomy 21 mosaic. Klin Padiatr 224(3):153-155

30. Gruber TA, Larson Gedman A, Zhang J, Koss CS, Marada S, Ta HQ et al (2012) An Inv(16)(p13.3q24.3)-encoded CBFA2T3GLIS2 fusion protein defines an aggressive subtype of pediatric acute megakaryoblastic leukemia. Cancer Cell 22(5):683-697

31. de Rooij JD, Hollink IH, Arentsen-Peters ST, van Galen JF, Berna Beverloo H, Baruchel A et al (2013) NUP98/JARID1A is a novel recurrent abnormality in pediatric acute megakaryoblastic leukemia with a distinct HOX gene expression pattern. Leukemia 27(12): 2280-2288

32. Karol SE, Coustan-Smith E, Cao X, Shurtleff SA, Raimondi SC, Choi JK et al (2015) Prognostic factors in children with acute myeloid leukaemia and excellent response to remission induction therapy. Br J Haematol 168(1):94-101 